\title{
THEORETICAL AND EXPERIMENTAL APPROACH TO TEST THE COHESION OF CALCIUM PHOSPHATE PASTES
}

\author{
M. Bohner ${ }^{1, *}$, N. Doebelin ${ }^{1}$ and G. Baroud ${ }^{2}$ \\ ${ }^{1}$ Dr hc Robert Mathys Foundation, Bischmattstrasse 12, 2544 Bettlach, Switzerland \\ ${ }^{2}$ Biomechanics Laboratory, Mechanical Engineering Department, Engineering Faculty, Université de Sherbrooke \\ Sherbrooke, Qc, Canada
}

\begin{abstract}
Recent studies have revealed that the ability of a calcium phosphate cement paste to harden in a physiological environment without desintegrating into small particles might be a key property to ensure a safe and reliable clinical use of calcium phosphate cements. However, this property called cohesion is not well understood and has not been studied extensively. The goal of the present study was to better understand which factors affect the cohesion of a calcium phosphate paste using the combination of a theoretical and experimental approach. In the theoretical approach, factors expected to influence the paste cohesion such as Van der Waals forces, electrostatic and steric interactions, as well as osmotic effects were listed and discussed. In the experimental approach, a new method to measure the cohesion of a non-setting calcium phosphate paste was presented and used to assess the effects of various factors on this property. The new method allowed a continuous measurement of cohesion and gave reproducible results. The experimental results confirmed the theoretical predictions: an increase of the liquid-to-powder ratio of the paste and of the powder particle size, as well as the addition of citrate ions and in limited cases dissolved xanthan polymer chains reduced the paste cohesion.
\end{abstract}

Key Words: Cohesion, calcium phosphate, cement, viscosity, paste, vertebroplasty, rheology

*Address for correspondence:

M. Bohner

Dr hc Robert Mathys Foundation

Bischmattstrasse 12

CH-2544 Bettlach

Switzerland

Telephone Number: +41326441413

Fax Number: +41326441176

E-mail: marc.bohner@rms-foundation.ch

\section{Introduction}

Osteoporosis has become in the last decades a major health problem for ageing societies. Highly osteoporotic bone is so brittle that any move can result in bone fracture. In 1995, 1.5 million fractures in the US were attributable to osteoporosis (Riggs and Melton, 1995). In 2000, 200’000 vertebral fractures were estimated to occur every year among the female US population (Melton and Kallmes, 2006). Even though these fractures are rather benign, they are associated with a high morbidity and long hospital stays (Lee and Yip, 1996). Repairing osteoporotic fractures with traditional osteosynthesis techniques is often very difficult due to the inherent weakness of bone. The introduction of screw-locking osteosynthesis plates has improved the therapy, but not in all indications. Therefore new techniques should be proposed, investigated and introduced.

One possibility is to reinforce bone by injecting a cementious bone substitute (Bohner et al., 1992). This technique is particularly useful for vertebral fractures in the so-called "vertebroplasty" (Galibert et al., 1987), during which a cement is injected into the vertebral body.

The main cement used in vertebroplasty is poly (methyl methacrylate) (PMMA). This cement presents several disadvantages such as monomer toxicity (Schoenfeld et al., 1979; Karlsson et al., 2005) and a large exothermicity of the setting reaction that may lead to bone necrosis (Deramond et al., 1997). A potential alternative to PMMA cement is represented by calcium phosphate cements (CPC) (Bai et al., 1999) These cements are biocompatible, and harden isothermally because the setting reaction occurs generally very slowly.

Unfortunately, CPC are poorly injectable (Bohner and Baroud, 2005) which often prevents their injection into osteoporotic bone. Moreover, the viscosity of CPC increases continually during setting (Baroud et al., 2004a) even though the viscosity should be constant and close to 100-1000 Pa during injection (Baroud et al., 2004b). Furthermore, recent studies have reported data suggesting thrombogenic properties of CPC (Bernards et al., 2004), possibly due to the release of calcium phosphate particles in the blood stream. Therefore, it is of great interest to control the rheological and aqueous properties of CPC.

In 1995, the first study was published on the use of small amounts of poly(saccharides) (e.g. chitosan or hydroxypropylmethyl cellulose) to improve the injectability of CPC (Andrianjatovo and Lemaître, 1995). The explanation for the latter effect was provided one decade later by Bohner and Baroud (2005) who used a theoretical approach to define the factors that control the cement injectability. Several factors were predicted to 
have a large importance, for example the average particle size, the particle size distribution, the use of flocculants and deflocculants, as well as the addition of lubricants. The effect of these factors was demonstrated in an experimental model in which non-reactive calcium phosphate particles ( $\beta$-tricalcium phosphate) were mixed with an aqueous solution, and the resulting paste was injected through a syringe and a cannula. Interestingly, the factors improving the cement injectability had different effects on the cement viscosity: whereas a decrease of the particle size or the addition of a lubricant (xanthan gel) increased the cement viscosity, the addition of poly(acrylic acid) had the opposite effect (Baroud et al., 2005).

CPC are made of calcium phosphate particles dispersed in an aqueous solution. During implantation, CPC pastes are meant to harden via crystal entanglement even though they are in an aqueous environment and hence could be dispersed and never harden. The ability of a hydraulic paste to harden in an aqueous environment without releasing loose particles has been termed "cohesion". So far, it is known that cohesion is improved with the addition of a polysaccharide hydrogel (Andrianjatovo and Lemaitre, 1995; Cherng et al., 1997; Ishikawa et al., 1995, 1997) or a decrease of the liquid-to-solid ratio (Khairoun et al., 1999). However, there is presently no understanding for these effects.

The goal of the present study is to define theoretically which factors are of importance for the cohesion of CPC and to demonstrate experimentally the validity of the theoretical approach.

\section{Theoretical}

The cohesion of a calcium phosphate cement paste must depend on the forces acting between the particles constituting the cement paste and on the interactions between the cement paste and the surrounding fluid. So, to determine the cohesion of a calcium phosphate cement paste, it appears essential to identify these forces and interactions, and later on to determine how these forces and interactions can be modified. This is the goal of the present section.

In general three main types of interaction energies are considered to take place between distinct particles present in a liquid, for example in a cement paste: (i) Van der Waals (attractive); (ii) electrostatic (repulsive); and (iii) steric (repulsive). The combined effects of van der Waals attraction and interparticle repulsion is represented by the Lennard-Jones equation (Hiemenz, 1986, p 620):

$$
\Phi=\xi x^{-12}-\beta x^{-6}
$$

in which $\Phi$ and $x$ are the force and the distance between two particles, and $\zeta$ and $\beta$ are two constants. This equation is valid for two identical molecules in vacuum, but similar relationships can be obtained between two particles in a liquid (approach of Hamaker (Ring, 1996)). However, the potential energy of attraction, $\Phi$, in a liquid decreases as the scale (particle size) increases (Hiemenz, 1986, p. 635):

$$
\Phi_{B} \propto \frac{1}{f} \Phi_{A}
$$

where the subscripts " $A$ " and " $B$ " refer to two particles sizes differing in size by a scaling factor " $f$ ". Noteworthy, the so-called "electrical double layer" (electrical charge profile at the particle surface) plays an important role in this approach. So, any change of the electrical double layer modifies the particle interactions and hence should modify the paste cohesion.

Often in ceramic processing, where the surface potential is small or the double layer thickness is thin, the electrostatic repulsion is not sufficient to stabilise the colloidal suspension against coagulation. As a result another form of stabilisation is needed - steric stabilisation. Basically, an adsorbed molecule, either a polymer (ionic or nonionic) or surfactant (typically ionic) with its associated solvent molecules can stabilise particle collisions because the adsorbed layer provides a steric hindrance to the close approach of the particles (Ring, 1996). There are two reasons for steric interactions: (i) a steric effect due to the fewer possible conformations of the adsorbed molecule in the region of the overlap, and (ii) the osmotic pressure effect due to the high concentration of chain elements in the region of the overlap. Steric stabilisation occurs when the polymer is either physically or chemically attached to the surface of the particles. Depletion stabilisation occurs when the polymer is free in solution and not adsorbed or chemically bound to the surface. Interestingly, steric repulsion does not depend on the nature of the underlying particle but depends only on polymer-polymer and polymer-solvent interactions.

Cohesion is not only a function of the interaction energies within the cement paste, but also a function of the interaction between the cement paste and the surrounding liquid. The latter interaction is mostly governed by the difference of osmotic pressure between cement interstitial fluid and surrounding liquid.

The osmotic pressure is given by the van't Hoff equation (Hiemenz, 1986, p. 121):

$$
n_{2}=\frac{\pi V_{1}}{R T}
$$

where $n_{2}$ is the number of moles of a solute in a solvent, $p$ is the osmotic pressure, $V_{1}$ is the volume of solvent in the solution, $T$ is the temperature and $R$ is the gas constant. This equation is valid for an ideal two-component solution, but similar relationships can be obtained in more complex systems. More details are given in Hiemenz (1986) and Ring (1996).

Now that the interaction energies occurring within the cement paste and between the cement paste and the surrounding fluid have been identified and described, it is of great interest to assess how the cohesion of the cement paste is affected by compositional effects.

Firstly, Van der Waals interaction energy depends on the distance between particles (" $x$ " in equation (1)). Therefore all factors that increase the interparticular distance such as an increase of the liquid-to-powder ratio 
Table 1: Summary of the effects expected to increase the cement paste cohesion.

\begin{tabular}{|l|l|}
\hline Increase of cohesion & How can it be achieved? $^{1}$ \\
\hline $\begin{array}{l}\text { Increase of...... } \\
\text {......Van der Waals interaction }\end{array}$ & $\begin{array}{l}\text { - Smaller particle size } \\
\text { - Shorter distance between the particles (e.g. lower L/P ratio, removal of all polymer molecules } \\
\text { present in the paste and/or at the particle surface) }\end{array}$ \\
\hline $\begin{array}{l}\text { Decrease of..... } \\
\ldots . . . \text {.electrostatic interactions }\end{array}$ & $\begin{array}{l}\text { - Ionic strength increase in the mixing liquid of the paste } \\
\text { - Desorption of adsorbed charged molecules (e.g. citrate ions) } \\
\text { - Shift of pH close to the isoelectric point }\end{array}$ \\
\hline $\begin{array}{l}\text { Decrease of.... } \\
\text {.....osmotic pressure }\end{array}$ & $\begin{array}{l}\text { - Decrease the concentration of dissolved species (e.g. ions, polymers) in the mixing liquid } \\
\text { - Increase the concentration of dissolved species (e.g. ions, polymers) in the incubation liquid }\end{array}$ \\
\hline $\begin{array}{l}\text { Decrease of.... } \\
\text {.......steric interactions }\end{array}$ & - Remove all polymer molecules present in the paste and/or at the particle surface \\
\hline
\end{tabular}

${ }^{1}$ In the sense that the interaction mentioned in the first column of the table is increased. For example, an increase of the Van der Waals interactions is expected to occur with a decrease of the average particle size.

${ }^{2}$ The pressure is considered positive when the osmotic pressure in the paste is larger than in the incubating solution.

(LPR) or the presence of adsorbed polymer chains at the particle surface decrease Van der Waals interaction energy. Moreover, equation (2) shows that the potential energy of attraction, $\Phi$, decreases as the scale increases. Therefore, the cohesion of a paste should decrease with an increase of particle size.

Secondly, electrostatic forces depend on the surface charge and the thickness of the electrical double layer. Thus all factors that decrease the surface charge or the thickness of the electrical double layer should increase cohesion. This can be achieved by different means. For example the surface charge can be decreased with a shift of the solution $\mathrm{pH}$ close to the isoelectric point of the particles in the solution ( $\mathrm{pH}$ at which the surface charge is null). The thickness of the electrical double layer can be decreased with an increase of the ionic force of the solution in which the particles are within (e.g. addition of disodium hydrogenophosphate typically used to accelerate the setting reaction of apatite cements). The adsorption of charged molecules (e.g. citrate ions or poly(acrylic acid)) at the particle surface leads to an increase of surface charge and hence decrease of cohesion.

Thirdly, steric stabilisation is typically related to the presence of a dissolved polymer at the surface of particles (when physically- or chemically-adsorbed) or in between them. Therefore, the addition of a polymeric rheological agent such as a polysaccharide gel (e.g., xanthan, sodium hyaluronate, hydroxypropylmethyl cellulose) or a carboxylated polymer (e.g., polyacrylic acid) is expected to sterically stabilise the cement paste (= prevent flocculation) and hence to decrease cohesion.

Finally, the osmotic pressure between the cement paste and the surrounding liquid depends on the difference in concentration of dissolved ions and molecules between the interstitial liquid of the cement paste and the surrounding liquid. In a simple approach if the total solute concentration in the solution is lower than in the interstitial liquid, the flow of molecules taking place from the solution into the cement will be larger than in the opposite direction. This flow results in the swelling of the cement surface, and hence to the decohesion of the cement. If the solute concentration in the solution is larger than in the interstitial liquid, the opposite is found: the cement surface tends to be depleted from its liquid, hence increasing cement cohesion. Therefore, the cohesion of a cement paste is expected to be better in serum (ionic strength close to $0.15 \mathrm{M}$ ) than in pure water. Moreover, an increase of the solute concentration in the interstitial liquid of the cement is expected to decrease cohesion. Examples are the addition of disodium hydrogenophosphate salt to accelerate the setting reaction (typical concentration close to $10^{-1} \mathrm{M}$ ), or the addition of polymeric rheological agents such as polysaccharide gels (e.g. xanthan, hydroxpropylmethyl cellulose, sodium hyaluronate) to improve cement injectability (typical concentrations close to $0.5-1.0 \mathrm{w} \%$ ). Noteworthy, the flow of species from the incubating liquid into the mixing liquid of the paste (or vice versa) is expected to proceed faster in a paste that has a high permeability. As permeability increases with an increase of particle size or liquid content, the osmotic pressure is expected to have a greater effect on the paste when the average particle size and the liquid-to-powder ratio are increased.

From the previous discussion, it appears that steric stabilisation occurs via a change of osmotic pressure when the added polymers are chemically or physically adsorbed on the particle surface. So the distinction between "steric stabilisation" and "osmotic pressure" is very confusing, because "steric stabilisation" and "osmotic pressure" refer in fact in this very particular case to the same thing. However, when polymers are used to sterically stabilise a powder without involving chemi- or physisorption, then the distinction between "steric stabilisation" and "osmotic pressure" is valid.

A summary of the previous considerations is given in Table 1. Interestingly, some factors are expected to have simultaneously a positive and a negative effect. For example, an increase of the ionic strength in the mixing liquid should increase cohesion due to a reduction of the thickness of the electrical double layer and hence reduction of the electrostatic interactions, but should also decrease cohesion due to an increase of the osmotic pressure. Table 
1 also reveals that the addition of an uncharged polymer into the mixing liquid should decrease cohesion due to a double effect: (i) increase of the osmotic pressure and (ii) steric stabilisation. Published scientific data has however shown that cohesion is increased with the addition of dissolved polysaccharides (Andrianjatovo and Lemaître, 1995; Cherng et al., 1997; Ishikawa et al., 1995, 1997). This points out the fact that other factors play a role on cement cohesion as well.

For example, it is difficult to prevent forces at the cement surface. These forces can be due to convection flow, pulsating blood or gravitation forces. The effect of these forces on cohesion is expected to decrease with an increase of the cement paste viscosity (e.g., achieved by adding dissolved polysaccharides in the mixing liquid). Brownian motion might also help decompose the cement surface, particularly for small particles. The StokesEinstein equation (Ring, 1996) predicts indeed that the diffusion coefficient is inversely proportional to the particle size. Noteworthy, cement cohesion is a transient property, so cement pastes with a very short setting time are expected to have a higher cohesion than slowly-setting cement pastes. It is therefore difficult to compare the cohesion of two cement pastes if their setting time is different. Furthermore, certain polymer gels like xanthan (Tempio and Zatz, 1980) can behave as flocculating agents by adsorbing simultaneously on several particles. A related mechanism is provided by sodium alginate which gels in the presence of divalent ions such as calcium ions (Levy and Schwarz, 1958). So when a sodium alginate solution is mixed with calcium phosphate powders (as in calcium phosphate cement formulations), sodium alginate rapidly reacts with the calcium ions released during the setting reaction to form a gel. This approach has been extensively described and used in the literature (Ishikawa et al., 1995, 1997).

\section{Experimental}

Test setup - Several methods have been proposed in the past to measure cohesion. Andrianjatovo and Lemaître (1995) injected the CPC paste into a Ringer solution, observed its stability visually, and measured the concentration of $\mathrm{Ca}$ ions released from the cement over time. Cherng et al. (1997) assessed the CPC paste resistance to washout in water by "hand scrubbing". The specimen were considered washout-proof when no visible surface abrasion resulted from scrubbing. Fernandez et al. (1994) proposed three methods to determine cohesion: (i) visual inspection; (ii) measurement of geometrical size of the cement; and (iii) a setting time test (Gilmore needle) to assess whether resoftening of the cement occurred. These authors concluded that the second method was not adequate. Khairoun et al. (1999) took over the first method proposed by Fernandez et al. (1994), i.e., performed a visual inspection of the cement surface after placing the cement in early contact with a Ringer solution. The socalled cohesion time was defined as the minimum time required to obtain a stable cement paste in solution. Ishikawa et al. $(1995,1997)$ proposed the only quantitative
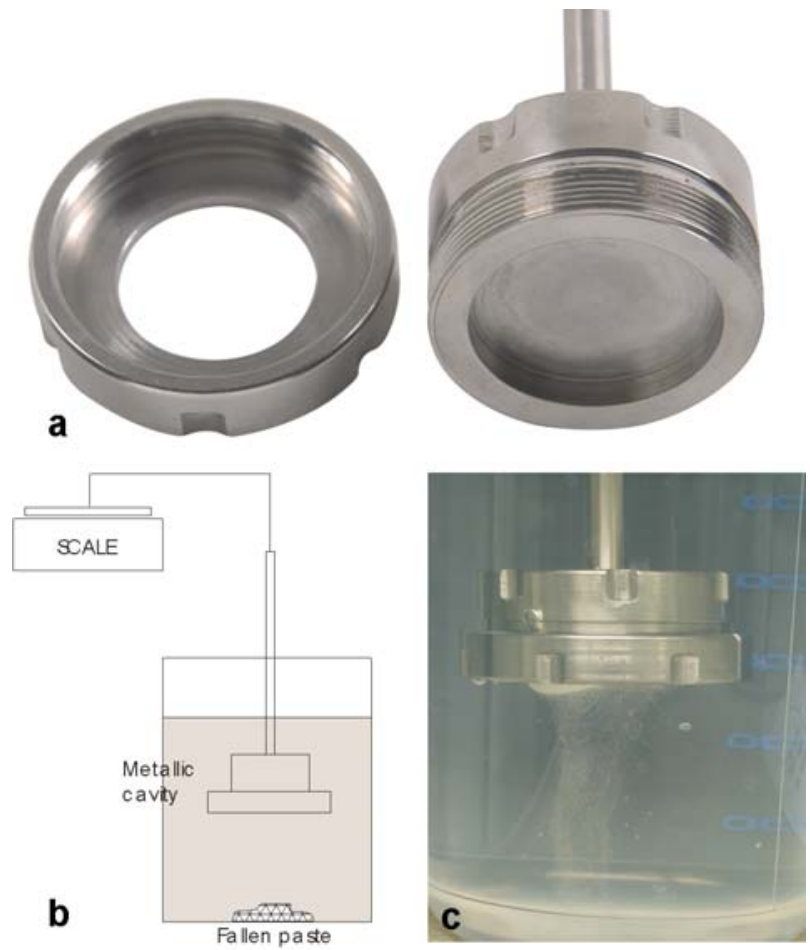

Figure 1: Experimental setup. (a) metallic cavity; (b) setup to measure the change of weight with the scale and the system to hold the metallic cavity; (c) small particles sedimenting from a $6 \mathrm{~g} \beta$-TCP powder $2.6 \mathrm{~mL} 0.6 \%$ xanthan solution mixture.

method: the weight fraction of cement still holding together after $24 \mathrm{~h}$ exposure to distilled water was determined.

The focus of the present study was to define factors affecting the cohesion of a paste independently of kinetic factors. In other words, the method had to be quantitative and applicable to non-setting pastes. Unfortunately, none of the methods published in the literature fulfilled both criteria (Andrianjatovo and Lemaître, 1995; Cherng et al., 1997; Fernandez et al., 1994; Ishikawa et al., 1995, 1997; Khairoun et al., 1999). Therefore, a new testing method was designed that allowed a continuous and quantitative assessment of the paste cohesion. For that purpose, the paste was suspended in a liquid and the weight of the suspended paste was recorded over time. In more detail, the paste was introduced into a metallic cavity of known dimension (diameter: $30 \mathrm{~mm}$; depth: $4 \mathrm{~mm}$ ) which could be partly closed with a metallic ring (Fig. 1a; diameter: $25 \mathrm{~mm}$; thickness: $2 \mathrm{~mm}$ ). This metallic cavity was then hooked onto a thread linked to an instrument lying on a scale (Fig. 1b). The metallic cavity was then lowered into the testing liquid in a slanted position to allow bubbles to escape from underneath the cement surface and the metallic cavity (Fig. 1c), and the weight of the construct was determined over time.

Sedimentation of the paste present in the metallic cavity provoked a decrease of weight measured on the scale. As the paste cohesion was defined as the ability of a hydraulic paste to harden in an aqueous environment without releasing loose particles, the paste cohesion was inversely proportional to the rate of weight loss. 
Table 2: List of experiments performed in this study. The liquid to powder ratio (LPR) is the ratio between the weight of liquid and the total weight of all solid components. Experiments were performed in d.i. water and in some cases in $4 \mathrm{w} \% \mathrm{NaCl}$ solution $^{(1)}$ and $0.1 \mathrm{M} \mathrm{Na}_{3} \mathrm{C}_{6} \mathrm{H}_{5} \mathrm{O}_{7} \cdot 2 \mathrm{H}_{2} \mathrm{O}$ solution ${ }^{(2)}$. Three to five experiments were performed for each composition.

\begin{tabular}{|c|c|c|}
\hline $\begin{array}{l}\text { Solid component of the } \\
\text { paste }\end{array}$ & $\begin{array}{l}\text { Composition of the } \\
\text { mixing liquid }\end{array}$ & $\begin{array}{l}\text { LPR } \\
{[\mathbf{m L} / \mathbf{g}]}\end{array}$ \\
\hline$\beta$-TCP powder $(6.00 \mathrm{~g})$ & $\begin{array}{l}\text { d.i. water } \\
10 \mathrm{w} \% \mathrm{NaCl} \\
0.6 \mathrm{w} \% \text { xanthan } \\
0.1 \mathrm{M} \mathrm{Na}_{3} \mathrm{C}_{6} \mathrm{H}_{5} \mathrm{O}_{7} \cdot 2 \mathrm{H}_{2} \mathrm{O}\end{array}$ & $\begin{array}{l}0.37,0.40,0.43,0.47, \text { and } 0.50 \\
(2.20,2.40,2.60,2.80,3.00 \mathrm{~mL}, \text { resp. }) \\
0.37,0.43 \text { and } 0.50 \\
0.37 \text { and } 0.43 \\
0.265^{(2)}\end{array}$ \\
\hline$\beta$-TCP granules $(6.00 \mathrm{~g})$ & d.i. water & $0.40(2.40 \mathrm{~mL})$ \\
\hline HA powder & $\begin{array}{l}\text { d.i. water } \\
0.6 \% \text { xanthan } \\
0.1 \mathrm{M} \mathrm{Na}_{3} \mathrm{C}_{6} \mathrm{H}_{5} \mathrm{O}_{7} \cdot 2 \mathrm{H}_{2} \mathrm{O}\end{array}$ & $\begin{array}{l}1.17,1.50, \text { and } 1.87 \\
(3.50,4.50,5.50 \mathrm{~mL} \text { for } 3.00 \mathrm{~g}, \text { resp. }) \\
1.50(3.00 \mathrm{~g} \text { powder for } 4.50 \mathrm{~mL}) \\
0.55(5.00 \mathrm{~g} \text { powder for } 2.75 \mathrm{~mL})^{(2)}\end{array}$ \\
\hline $\begin{array}{l}\beta \text {-TCP powder }(6.00 \mathrm{~g})+ \\
\beta \text {-TCP granules }(2.00 \mathrm{~g})\end{array}$ & $\begin{array}{l}\text { d.i. water } \\
0.6 \mathrm{w} \% \text { xanthan }\end{array}$ & $\begin{array}{l}0.275,0.325, \text { and } 0.375^{(1)} \\
(2.20,2.60, \text { and } 3.00 \mathrm{~mL}) \\
0.325(2.60 \mathrm{~mL})\end{array}$ \\
\hline $\begin{array}{l}\beta \text {-TCP powder }(6.00 \mathrm{~g})+ \\
\text { HA powder }(0.5 \mathrm{~g})\end{array}$ & d.i. water & $0.40(2.60 \mathrm{~mL})$ \\
\hline
\end{tabular}

\section{Materials and methods}

Three powder/granule types were used: (i) $\beta$-Tricalcium phosphate (micro) powder ( $\beta$-TCP; Fluka No 21218, 455807/1, 31404044; Buchs, Switzerland). This powder has a mean volume diameter close to $10 \mu \mathrm{m}$ (Baroud et al., 2004b), and a specific surface area (SSA) of $1.10 \mathrm{~m}^{2} / \mathrm{g}$ (Standard deviation (SD): $0.01 \mathrm{~m}^{2} / \mathrm{g}$ ). According to $\mathrm{x}$-ray diffraction (XRD), it contains a small amount $(<5 \%)$ of $\beta$ calcium pyrophosphate $\left(\mathrm{Ca}_{2} \mathrm{P}_{2} \mathrm{O}_{7}\right)$ (Fig. 2). (ii) Hydroxyapatite (nano) powder (HA; Merck No 1.02196, 225K17691496; Darmstadt, Germany). This powder has an SSA of $62.53 \mathrm{~m}^{2} / \mathrm{g}\left(\mathrm{SD}=0.20 \mathrm{~m}^{2} / \mathrm{g}\right)$ and is according to XRD phase pure, but poorly crystalline (broad peaks). (iii) round $\beta$-TCP granules (produced by Brace $\mathrm{GmbH}$, Alzenau, Germany; diameter $0.3 \pm 0.1 \mathrm{~mm}$ ). These granules have a packing density of $1.66 \mathrm{~g} / \mathrm{cc}$. The morphology of these different powders/granules is represented in Fig. 3. A large number of experiments was performed (Table 2). Factors of interest were: (i) The composition of the solid component of the paste (powder, granule or mixture); (ii) The composition of the mixing liquid; (iii) The Liquid-toPowder Ratio (LPR); and (iv) The composition of the incubating solution. The chemicals used for these experiments were (i) Sodium chloride $(\mathrm{NaCl}$; Merck No 1.06400, 31510100 249); (ii) Tri-sodium citrate dihydrate $\left(\mathrm{Na}_{3} \mathrm{C}_{6} \mathrm{H}_{5} \mathrm{O}_{7} \cdot 2 \mathrm{H}_{2} \mathrm{O}\right.$; Merck No 1.06448, A118748 909, 31.05.01; Buchs); and (iii) xanthan (Jungbunzlauer,

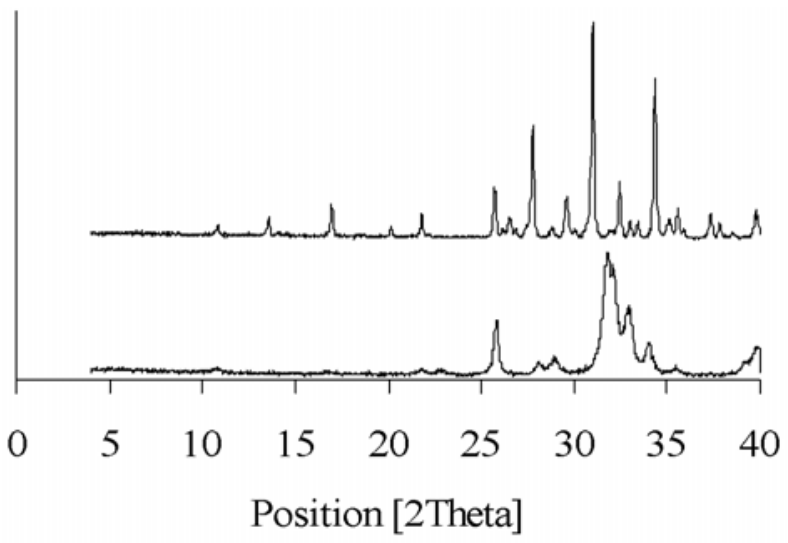

Figure 2: XRD data of HA powder (Bottom) and $\beta$ TCP (Top). HA powder is phase pure, but poorly crystalline (broad peaks). $\beta$-TCP contains small $\beta$ $\mathrm{Ca}_{2} \mathrm{P}_{2} \mathrm{O}_{7}$ impurities, but is well crystallized.

Pernhofen, Austria; xanthan solution contained $0.02 \mathrm{w} \%$ $\mathrm{NaN}_{3}$ to prevent its deterioration). Three to five measurements were performed for each composition. The weight was recorded every 5-15s using a Mettler Toledo (Columbus, OH; USA) Scale PR5002 coupled to a computer (Software: Balance Link 4.0.0).

The preparation of the paste was the following: the required amounts of solid and liquid were mixed with a metallic spatula in a glass beaker for 30 seconds. 

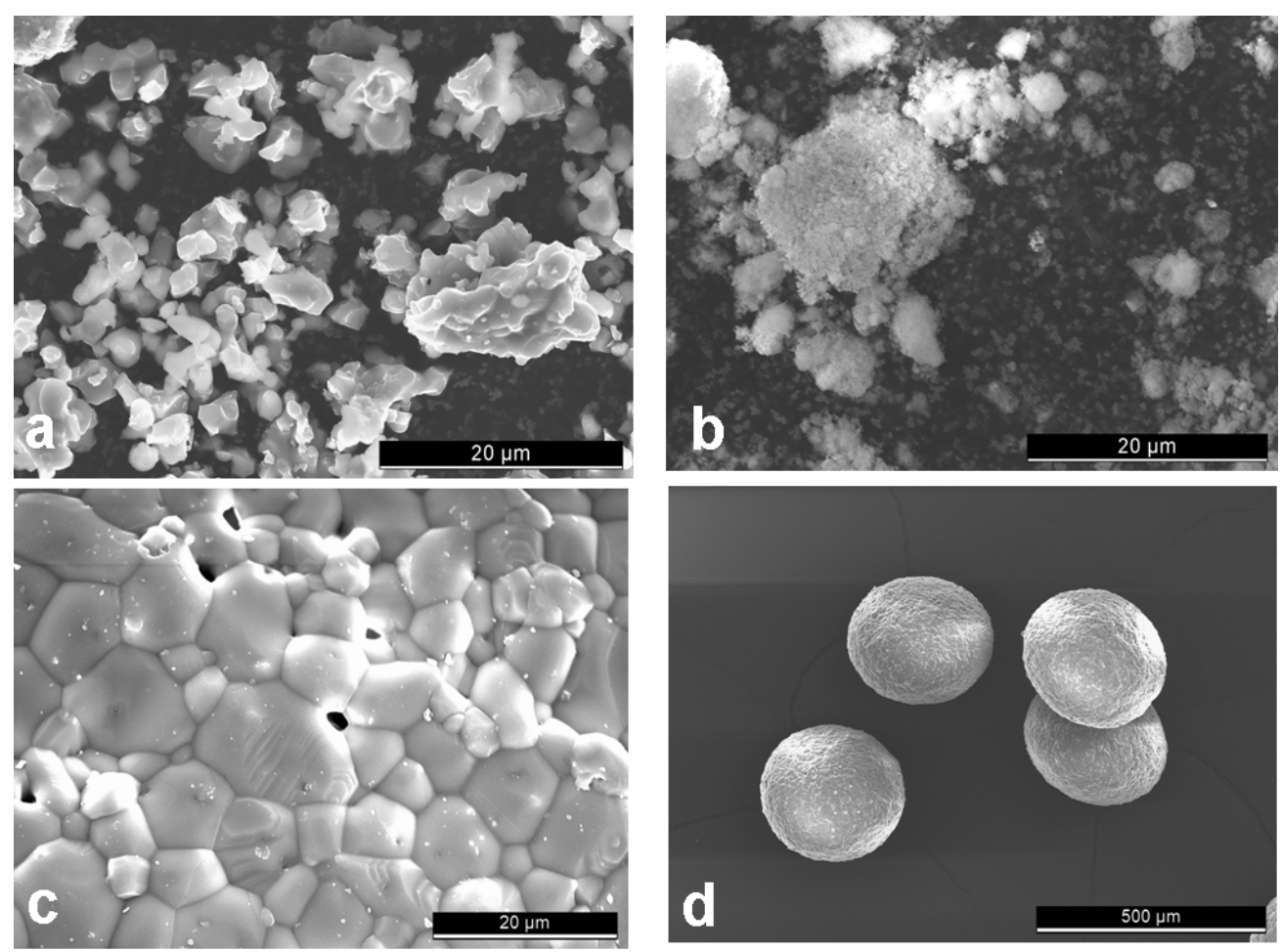

Figure 3: Morphology of the three powders/granules used in this study. (a) $\beta$-TCP powder (scale bar: $20 \mu \mathrm{m})$; (b) HA powder (scale bar: $20 \mu \mathrm{m}$ ); (c) $\beta$-TCP granules (scale bar: $20 \mu \mathrm{m}$ ); and (d) $\beta$-TCP granules (scale bar: $500 \mu \mathrm{m}$ ). The HA powder consists of agglomerates of tiny crystals that can be hardly distinguished on the present SEM photo. The low electrical conductivity makes it difficult to obtain a better enlargement (even after glueing the powder on a conductive tape and gold sputtering it).

Simultaneously, the glass beaker was vibrated on a Vortex (Vortex Genie 2, Scientific Industries, Bohemia, NY, USA). The paste was then placed into the metallic cavity using the spatula, the metallic ring was screwed onto the metallic cavity, and the whole was vibrated on the Vortex (vertically, cement on top) to remove air bubbles and cement heterogeneities. Vortexing was stopped when the paste surface remained smooth (typically after 30-60 seconds). Pastes containing citrate ions had to be mixed for 23 minutes due to the fact that it took a long time for the powder to be wetted by the liquid.

\section{Results}

Curves obtained with mixtures of deionized water and $\beta$ TCP powder were typically characterized by an absence of weight change (no particle release) followed by a rapid drop of weight and again a weight plateau (Fig. 4a). The time at which the whole paste fell was defined as the "drop time". This drop time increased with a decrease of LPR. HA-based pastes behaved differently, i.e. several weight changes were observed along the curve, particularly at large LPR. This prevented the definition of a drop time. Interestingly, the stability of the pastes varied markedly depending on the nature of the calcium phosphate powder: typically, the drop time was in the range of 5-20 minutes with a microsized $\beta$-TCP powder (Fig. 4a), whereas pastes based on HA powder were still falling down after a few hours (Fig. 4b). When a small amount of HA was added to a paste containing $\beta$-TCP powder and d.i. water, the drop time increased markedly (Fig. 4a). Finally, the drop time of a paste consisting of $6 \mathrm{~g}$ round $\beta$-TCP granules and $2.4 \mathrm{~mL}$ d.i. water was shorter than 1 second (not represented on Fig. 4).

Additives present in the mixing solution had generally a large effect on the paste cohesion. For example, in the presence of a $0.1 \mathrm{M}$ citrate solution, the LPR had to be reduced to $0.265 \mathrm{~mL} / \mathrm{g}$ with $\beta$-TCP and $0.546 \mathrm{~mL} / \mathrm{g}$ with HA to prevent the paste from flowing out of the cavity. Additionally, a slow but continuous decrease of weight was observed (Fig. 5). Interestingly, the rate of weight loss was larger in d.i. water than in $0.1 \mathrm{M}$ citrate solution.

Mixtures of $\beta$-TCP powder and xanthan solution were characterised by a slow but continuous weight loss and particle release (Fig. 6). Replacing $\beta$-TCP with HA led to a complete absence of particle release and weight loss, but the paste swelled noticeably. The weight of the paste increased by $0.97 \mathrm{~g}(\mathrm{SD}=0.05 \mathrm{~g})$ during the course of three performed measurements.

As previously mentioned, adding HA powder to a $\beta$ TCP powder - d.i. water mixture markedly increased the paste cohesion (Fig. 4). The opposite effect was observed while blending $\beta$-TCP powder with $\beta$-TCP granules (Fig. 7). Interestingly, the loss of weight was not incidental but continuous. In fact, the absolute weight loss rate tended to increase throughout the experiment and the paste stability was reduced with an increase of LPR. Performing the test in $4 \mathrm{w} \% \mathrm{NaCl}$ solution slightly increased the drop time (Fig. 7,8), but the effect was not significant. A much more pronounced stabilisation effect was observed when xanthan was added into $\beta$-TCP powder $-\beta$-TCP granules - water mixtures (Fig. 7). 

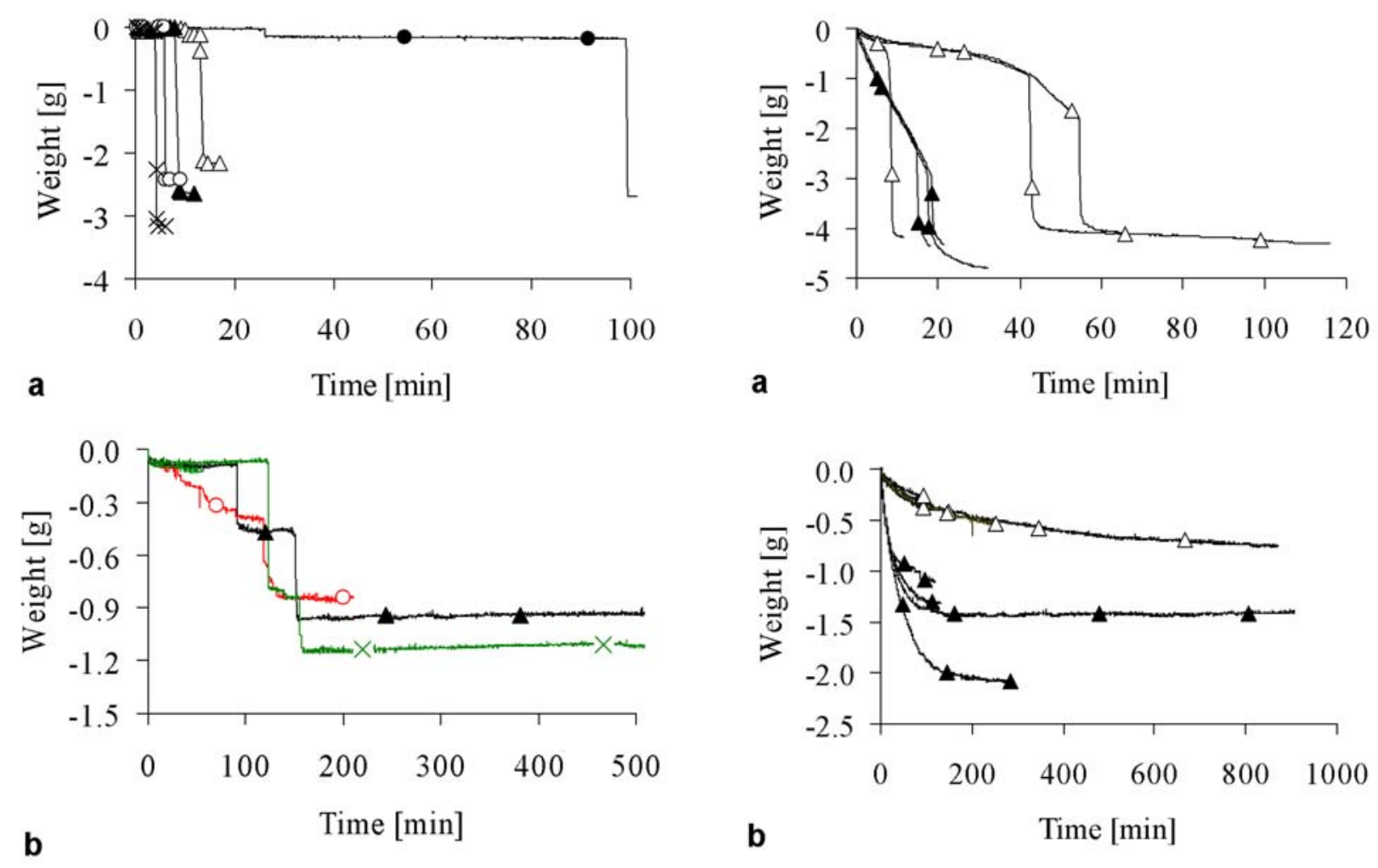

Figure 4: Typical curves obtained with the cohesion test. Compositions in (a): $6.00 \mathrm{~g} \beta$-TCP powder and (x) $3.00 \mathrm{~mL}$; (o) $2.80 \mathrm{~mL}$; ( $\Delta$ ) $2.60 \mathrm{~mL}$; $\triangle$ ) $2.40 \mathrm{~mL}$ d.i. water; (O) $6.00 \mathrm{~g} \beta$-TCP powder, $0.50 \mathrm{~g}$ HA powder, and $2.60 \mathrm{~mL}$ d.i. water. Composition in (b): $3 \mathrm{~g}$ HA powder and (x) $3.50 \mathrm{~mL}$; ( $\mathbf{\Delta}) 4.50 \mathrm{~mL}$; (o) $5.50 \mathrm{~mL}$ d.i. water. All measurements were made in d.i. water. Only one representative measurement is shown for clarity reasons.

Looking at the drop time values (Fig. 8), it appears that the main effects were due to a change of LPR or to the presence of $\beta$-TCP granules. The addition of $\mathrm{NaCl}$ in the mixing or in the incubation liquid did not significantly influence the results.

\section{Discussion}

The various powders, granules and additives used in the present study have been selected because all have been used in the composition of calcium phosphate cements. For example, $\beta$-TCP powder and granules have been used to obtain a so-called brushite cement (Ohura et al., 1996), whereas hydroxyapatite powder is often used in the composition of apatite cements to accelerate the setting reaction (Chow, 1996). Furthermore, additives such as citrate ions (Bohner et al., 1996), and xanthan (Lu et al., 1999) have been described in combination with calcium phosphate cements.

Two mechanisms of cohesion loss were observed: either a continuous but slow process as seen with HA powder and a high LPR (Fig. 4b), citrate ions (Fig. 5), xanthan (Fig. 6), and $\beta$-TCP granules (Fig. 7), or a rapid weight loss as seen for example with $\beta$-TCP - d.i. water mixtures (Fig. 4). In both cases, the weight loss was due to the action of gravity forces on the paste. However, the detailed mechanism of action was different. In the former case, the paste lost its cohesion at the microscopic scale, i.e. continuously released small units formed of a few particles/granules (Fig 1c). In the latter case, the paste lost cohesion at the macroscopic scale, i.e. the whole paste fell down at once. This effect was only possible if the paste changed in such a way that the shear forces at the metal-paste interface became suddenly larger (at the drop time) than the sum of the paste cohesion and the paste adhesion to the metallic cage. The absence of visible and measurable paste swelling suggests that the latter effect was not related to an increase of LPR during the measurement, but to a change of the paste rheological properties at the metal-paste interface.

The setup to test the paste cohesion has proved to work well. Contrary to previously-described methods in the literature, this method is continuous which enables to determine the time evolution of cohesion. The method is also independent of the testing person. However, the occurrence of a discrete phenomenon (paste drop) underlines the fact that the test method cannot be applied to flowing pastes. Moreover, the test setup depends on gravity forces which are not exactly the same as the convection forces typically found at the cement-blood surface during implantation. Regarding this matter, the 


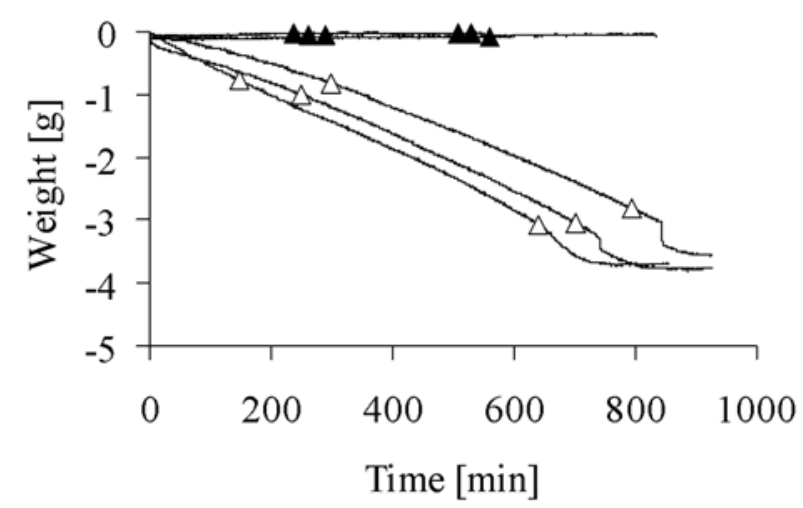

Figure 6: Slow sedimentation with a $0.6 \mathrm{w} \%$ xanthan solution (with $0.02 \% \mathrm{NaN}_{3}$ ); $\triangle(\triangle) 6.00 \mathrm{~g} \beta$-TCP powder and $2.6 \mathrm{~mL}$ solution, and $(\boldsymbol{\Delta}) 3 \mathrm{~g}$ HA powder and $4.5 \mathrm{~mL}$ solution. The incubation solution was d.i. water.

cohesion test setup allows some stirring during the measurement, but intensive stirring leads to strong fluctuations in weight measurement and hence prevents an accurate continuous assessment of paste cohesion.

A large range of parameters have been identified theoretically to have an effect on the paste cohesion. Such parameters included the particle size and the presence of various additives in the mixing liquid. Experimental results confirmed most predictions of the theoretical approach: the paste cohesion was reduced with large particles (Fig. 4-7), and a high LPR (Fig. 7), as well as with the addition of citrate ions (Fig. 5) and in limited cases with xanthan (Fig. 6).

The effect of xanthan was rather complex since xanthan addition decreased the cohesion of $\beta$-TCP - water mixtures but improved the cohesion of HA - water mixtures (Fig. 6). In the latter case, an important swelling of the paste was noticed. Moreover, the weight of the paste was strongly increased during the course of the experiment $(0.97 \mathrm{~g} ; \mathrm{SD}=0.05 \mathrm{~g})$. This effect is likely to be due to the osmotic pressure provoked by the presence of xanthan in the mixing liquid of the paste. So, apparently the osmotic pressure was not large enough in HA-based pastes to lead to a loss of cohesion, contrary to $\beta$-TCP-based pastes. Interestingly, past studies have demonstrated a positive effect of hydrogels such as xanthan on the cohesion of calcium phosphate cement paste (Andrianjatovo and Lemaître, 1995; Cherng et al., 1997; Ishikawa et al., 1995, 1997). So apparently, calcium phosphate cement pastes react like HA-based pastes rather than like $\beta$-TCP-based pastes.

Pastes obtained with blends of $\beta$-TCP powder and granules presented an increasingly fast weight loss rate (Fig. 7). This effect could be due to the mode of paste preparation. The paste was indeed vibrated for 30 seconds before testing, which may have led to the sedimentation of the granules within the paste, and hence to a gradient of paste cohesion. Unfortunately, it was not possible to observe a gradient of granule concentration to support the present explanation.

The weight loss rate of pastes obtained with a $0.1 \mathrm{M}$ citrate solution tended to decrase over time with HA powder and to increase over time with $\beta$-TCP powder (Fig.

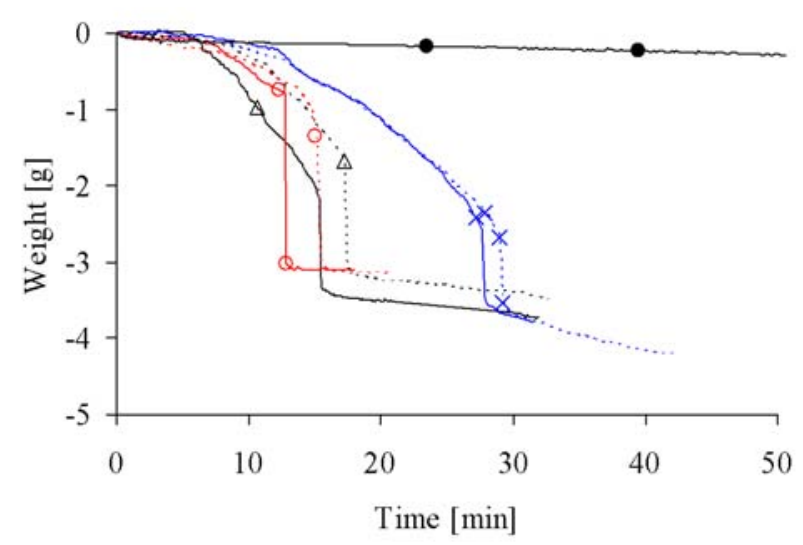

Figure 7: Change of weight for $\beta$-TCP powder + granule mixtures $(6.00 \mathrm{~g}$ powder $+2.00 \mathrm{~g}$ granules $)$. Incubation in d.i. water (plain line) or $4 \mathrm{w} \% \mathrm{NaCl}$ solution (dotted line). Amount and nature of the mixing solution: $(\mathrm{x}) 2.2 \mathrm{~mL}$ d.i. water; $(\triangle) 2.6 \mathrm{~mL}$ d.i. water; (o) $3.0 \mathrm{~mL}$ d.i. water; (○) $2.6 \mathrm{~mL} 0.6 \mathrm{w} \%$ xanthan solution. For clarity reasons, only one representative curve is shown per composition.

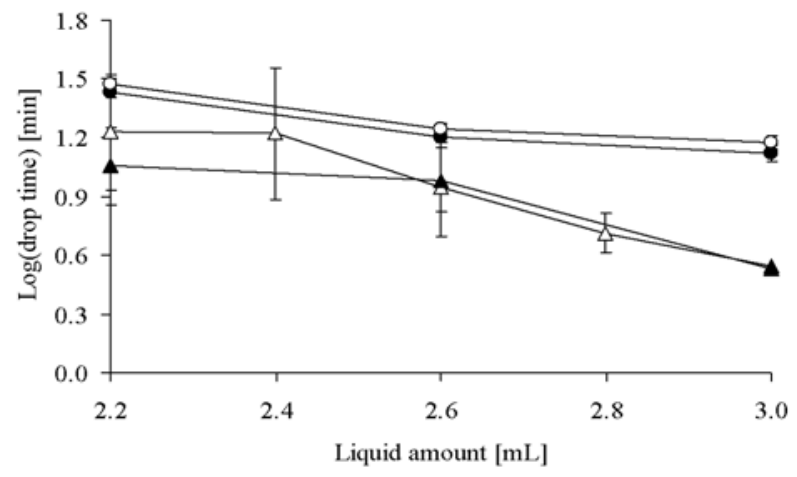

Figure 8: Change of cohesion time as a function of the $\mathrm{L} / \mathrm{P}$ ratio. $(\triangle) 6.00 \mathrm{~g} \beta$-TCP powder and d.i. water in d.i. water; $(\mathbf{A}) 6.00 \mathrm{~g} \beta$-TCP powder and $10 \mathrm{w} \%$ $\mathrm{NaCl}$ solution in d.i. water; $(\mathbf{O}) 6.00 \mathrm{~g} \beta$-TCP powder, $2.00 \mathrm{~g} \beta$-TCP granules, and d.i. water and d.i. water; (o) $6.00 \mathrm{~g} \beta$-TCP powder, $2.00 \mathrm{~g} \beta$-TCP granules, and d.i. water in $4 \mathrm{w} \% \mathrm{NaCl}$ solution. The error bar corresponds to \pm one standard deviation.

6). This difference is puzzling and cannot be explained at present. Nevertheless, it is worth mentioning that both pastes showed a strong shear thinning behaviour and had to be vibrated extensively ( $>1$ minute) before testing, hence possibly resulting in a composition gradient within the paste.

The paste cohesion was not only a function of the composition of the paste, but also of the incubating solution, as observed with a $0.1 \mathrm{M}$ citrate solution (Fig. 5). The later result can be explained with a change of osmotic pressure between the incubating solution and mixing solution (Table 1). However, it is not clear why the effect of the citrate solution was so large whereas the effect of the $\mathrm{NaCl}$ solution was not significant.

Present results suggest that the two main methods to increase the cohesion of a calcium phosphate (cement) paste are to decrease the average particle size of the paste 
constituents (milling the powder more intensively or adding a finer powder - Fig. 4), and in some cases to add xanthan. Interestingly, both approaches are known to improve the paste injectability (Bohner and Baroud, 2005).

\section{Conclusion}

Various parameters potentially affecting the cohesion of a calcium phosphate (cement) paste have been identified theoretically and tested using a new method. The test method can be used for non cementitious and non flowable pastes and provides reliable and reproducible data. Most effects predicted in the theoretical approach could be confirmed experimentally. These effects included a change of liquid-to-powder ratio (LPR), of particle size, of composition of the mixing liquid (citrate or in some cases xanthan addition) and of the incubating solution $(\mathrm{NaCl}$ or citrate addition). The results suggest that the two best methods to increase the cohesion of a calcium phosphate paste are to decrease the average particle size of the paste constituents or in some cases to add a hydrogel such as xanthan.

\section{References}

Andrianjatovo H, Lemaître J (1995) Effects of polysaccharides on the cement properties in the monocalcium phosphate/b-tricalcium phosphate system. Innov Tech Biol Med 16(S1): 141-147.

Bai B, Jazrawi LM, Kummer FJ, Spivak JM (1999) The use of an injectable, biodegradable calcium phosphate bone substitute for the prophylactic augmentation of osteoporotic vertebrae and the management of vertebral compression fractures. Spine 24: 1521-1526.

Baroud G, Matsushita C, Samara M, Beckman L, Steffen T (2004a) Influence of oscillatory mixing on the injectability of three acrylic and two calcium-phosphate bone cements for vertebroplasty. J Biomed Mater Res 68B: 105-111.

Baroud G, Bohner M, Heini P, Steffen T (2004b) Injection biomechanics of bone cements used in vertebroplasty. Biomed Mater Eng 14: 487-504.

Baroud G, Cayer E, Bohner M (2005) Rheological characterization of concentrated aqueous b-tricalcium phosphate suspensions: the effect of liquid-to-powder ratio, milling time and additives. Acta Biomaterialia 1: 357-363.

Bernards CM, Chapman JR, Mirza SK (2004) Lethality of embolized norian bone cement varies with the time between mixing and embolization. Proc 50th Annual Meeting of the Orthopaedic Research Society, San Francisco, USA, p 254.

Bohner M, Baroud G (2005) Injectability of calcium phosphate pastes. Biomaterials 26: 1553-1563.

Bohner M, Lemaitre J, Cordey J, Gogolewski S, Ring TA, Perren SM (1992) Potential use of biodegradable bone cement in bone surgery: holding strength of screws in reinforced osteoporotic bone. Orthopaedic Trans 16: 401402.

Bohner M, Lemaitre J, Ring TA (1996) Effects of sulfate, pyrophosphate, and citrate ions on the physicochemical properties of cements made of betatricalcium phosphate-phosphoric acid-water mixtures. J Am Ceram Soc 79: 1427-1434.

Cherng A, Takagi S, Chow LC (1997) Effects of hydroxypropyl methylcellulose and other gelling agents on the handling properties of calcium phosphate cement. J Biomed Mater Res 35: 273-277.

Chow LC (1991) Development of self-setting calcium phosphate cements. J Ceram Soc Japan 99: 954-964.

Deramond H, Depriester C, Toussaint P, Galibert P (1997) Percutaneous Vertebroplasty. Semin Musculoskelet Radiol 1: 285-296.

Fernandez E, Boltong MG, Driessens FCM, Ginebra MP, Bermudez O, Planell JA (1994) The swelling of calcium phosphate cements. Proc $11^{\text {th }}$ European Conference on Biomaterials, Pisa, Italy, p 314.

Galibert P, Deramond H, Rosat P, Le Gars D (1987) [Preliminary note on the treatment of vertebral angioma by percutaneous acrylic vertebroplasty]. Neurochir 33: 166-168.

Hiemenz PC (1986) Principles of colloid and surface chemistry. $2^{\text {nd }}$ ed, Dekker, New York, USA.

Ishikawa K, Miyamoto Y, Kon M, Nagayama M, Asaoka K (1995). Non-decay type fast-setting calcium phosphate cement: composite with sodium alginate. Biomaterials 16: 527-532.

Ishikawa K, Miyamoto Y, Takechi M, Toh T, Kon M, Nagayama M, Nagayama M, Asaoka K (1997) Non-decay type fast-setting calcium phosphate cement: hydroxyapatite putty containing an increased amount of sodium alginate. J Biomed Mater Res 36: 393-399

Karlsson J, Wendling W, Chen D, Zelinsky J, Jeevanandam V, Hellman S, Carlsson C (1995) Methylmethacrylate monomer produces direct relaxation of vascular smooth muscle in vitro. Acta Anaesthesiol Scand 39: 685-689.

Khairoun I, Driessens FCM, Boltong MG, Planell JA, Wenz R (1999) Addition of cohesion promotors to calcium phosphate cements. Biomaterials 20: 393-398.

Lee YL, Yip KMH (1996) The osteoporotic spine. Clin Orthopaedic Rel Res 323: 91-97.

Levy G, Schwarz TW (1958) The role of residual calcium in the viscosity changes of sodium alginate solutions. J Am Pharm Assoc Am Pharm Assoc (Baltim) 47: 455-457.

Lu JX, About I, Stephan G, Van Landuyt P, Dejou J, Fiocchi M, Lemaitre J, Proust JP (1999) Histological and biomechanical studies of two bone colonizable cements in rabbits. Bone 25(2 Suppl): 41S-45S.

Melton LJ, Kallmes DF (2006) Epidemiology of vertebral fractures: implications for vertebral augmentation. Acad Radiol 13: 538-545.

Ohura K, Bohner M, Hardouin P, Lemaître J, Pasquier G, Flautre B (1996) Resorption of, and bone formation from, new $\beta$-tricalcium phosphate-monocalcium phosphate cements: an in vivo study. J Biomed Mater Res 30: 193 200.

Riggs BL, Melton LJ (1995) The worldwide problem of osteoporosis: insights afforded by epidemiology. Bone 17: 505S-511S. 
Ring TA (1996) Fundamentals of ceramic powder processing and synthesis. Academic Press, San Diego, USA.

Schoenfeld CM, Conard GJ, Lautenschlager EP (1979) Monomer release from methacrylate bone cements during simulated in vivo polymerization. J Biomed Mater Res 13: $135-147$.

Tempio JS, Zatz JL (1980) Flocculation effect of xanthan gum in pharmaceutical suspensions. J Pharm Sci 69: $1209-1214$.

\section{Discussion with Reviewers}

J.D. de Bruijn: The authors mention that one of the reported risks with non-hardened or non-cohesive calcium phosphate cement (CPC) pastes is the occurrence of thrombosis due to the release of calcium phosphate particles in the blood stream. The proposed test will evaluate the cohesion of the cement which might therefore reduce this risk. However, it does not take cement degradation into consideration which may also result in small calcium phosphate particles. Can the authors comment on this possible longer-term risk of degrading CPC pastes?

Authors: The long-term release of calcium phosphate particles has been a matter of concern for us after reading the report of Bernards et al. (2004), particularly because none of us is an expert in haematology. To improve our understanding of the problem, we initiated a study with a haematologist and we tried to contact various experts. Our present understanding is that the risk of clotting related to the release of particles after the degradation/resorption of calcium phosphate cements is small, if not negligible. A few hours after implantation, a calcium phosphate cement is surrounded by a blood clot. At later time points, the cement is surrounded by soft tissues or bone, and particles tend to be phagocytosed. As a result, particle release into the blood stream is very unlikely to happen, at least not in the extent seen during implantation.

It has to be mentioned that blood vessels in vertebral bodies are apparently not really "closed" vessels as in most other locations in the body. It is more like a sponge. As a result, the injection of a calcium phosphate cement paste injected close to the blood vessel can release particles in large amounts.

Clinically, there is to our knowledge no report relating thrombo-embolism and calcium phosphate degradation. Noteworthy, Professor M.A. Rauschmann (Frankfurt) reported at GRIBOI 2005 (Shanghai) 2 cases of death due to thrombo-embolism one month after the implantation of "Osteoset pellets" (calcium sulfate dihydrate; CSD). However, M.A. Rauschmann mentioned that a link between thrombo-embolism and the implantation of CSD could be excluded.

R. Oreffo: From a clinical perspective what are the limitations to the use of xanthan as advocated in the current paper?
Authors: The current limitation in the use of xanthan clinically is its purity and perhaps lack of use. The products used for pharmaceutical applications (topical applications) and in the food business are not pure enough to be implanted. Implantation of such products would lead to biocompatibility problems. The effect of impurities on the in vivo response is a well-understood problem of hyaluronan products.

R. Oreffo: Have you considered the use of collagen/ hydrogels in very dilute concentrations to aid cohesiveness of milled powders -is there any rationale for not adopting such a strategy?

Authors: The use of human-derived products such as collagen is not an option at Mathys Foundation due to philosophical reasons. Recombinant collagen could be considered, but its price is very high and its availability is very limited. The product we typically use is sodium hyaluronate from recombinant origin.

R. Oreffo: The use of the displacement rod apparatus is intriguing - does increased weight at swelling result in skew of data sets and how is this compensated?

Authors: The exchange of liquid between the mixing liquid of the paste and the incubating solution can only skew the data if the density of the two liquids is not identical. As only aqueous solutions were used with densities close to 1.0, data skewing can be neglected. In more details, the weight measured by the scale is the combination of the weight of the apparatus filled with the cement paste minus the upwards force generated by the displacement of incubating liquid (Archimedes force). If the cement paste absorbs liquid (e.g. $1 \mathrm{~mL}$ ), the apparatus becomes heavier (i.e. about $1 \mathrm{~g}$ for $1 \mathrm{~mL}$ - assuming that water is absorbed), but the Archimedes force is simultaneously increased (i.e. about $1 \mathrm{~g}$ for $1 \mathrm{~mL}$ ). The net change of weight is close to zero.

R. Oreffo: In figure 5, the sedimentation curves appear highly variable. Could you give a mean summation?

Authors: We do not think it makes sense to group the curve because a mean curve (mean of three curves) would show some drops along the curves only because 1 out of 3 curves dropped. This would give a false idea of the experimental behavior. Additionally, we would have a problem to obtain a mean curve over the overall duration since the measurements were not performed for the same duration. Basically, the experiments were stopped when the main weight drop had occurred or when a stable behavior had been reached. Here, we decided to only show one representative curve per composition.

\section{Additional Reference}

Bernards CM, Chapman JR, Mirza SK (2004) Lethality of embolized norian bone cement varies with the time between mixing and embolization. Proc 50th Annual Meeting of the Orthopaedic Research Society, San Francisco, p. 254. 